\title{
Object Based and Pixel Based Classification using Rapideye Satellite Imagery of Eti-Osa, Lagos, Nigeria
}

\author{
E. O. Makinde ${ }^{\text {a }}$, A. T. Salami ${ }^{\mathrm{b}}$, J. B. Olaleye ${ }^{\mathrm{a}}$, O. C. Okewusi ${ }^{\mathrm{a}}$ \\ ${ }^{a}$ Department of Surveying and Geoinformatics, \\ Faculty of Engineering, University of Lagos, \\ Akoka, Lagos, Nigeria \\ b Space Applications and Environmental Science Laboratory, \\ Institute of Ecology and Environmental Studies, \\ Faculty of Science, Obafemi Awolowo University, \\ Ile-Ife, Osun, Nigeria \\ estherdanisi@gmail.com, ayobasalami@yahoo.com, jbolaleye@yahoo.com, \\ pelcool55@yahoo.com
}

\begin{abstract}
Several studies have been carried out to find an appropriate method to classify the remote sensing data. Traditional classification approaches are all pixel-based, and do not utilize the spatial information within an object which is an important source of information to image classification. Thus, this study compared the pixelbased and object-based classification algorithms using RapidEye satellite image of Eti-Osa LGA, Lagos. In the object-oriented approach, the image was segmented to homogenous areas by suitable parameters such as a scale parameter, compactness, shape etc. Classification based on segments was done by a nearest neighbour classifier. In the pixel-based classification, the spectral angle mapper was used to classify the images. The user accuracy for each class using object-based classification were $98.31 \%$ for water body, 92.31\% for vegetation, $86.67 \%$ for bare soil and 90.57\% for built up areas while the user accuracy for the pixel-based classification were $98.28 \%$ for water body, $84.06 \%$ for vegetation $86.36 \%$ and $79.41 \%$ for built up areas. These classification techniques were subjected to accuracy assessment and the overall accuracy of the object-based classification was $94.47 \%$, while that of pixel-based classification yielded $86.64 \%$. The results of classification and its accuracy assessment show that the object-based approach gave more accurate and satisfying results.
\end{abstract}

Keywords: RapidEye satellite image; pixel-based classification; object-based classification.

\section{Introduction}

According to the findings of [2], geospatial specialists have theorized the possibility of developing a fully automated classification procedure that would be an improvement over pixel-based procedures. Pixel-based procedures analyse the spectral properties of every pixel within an area of interest, without taking into account the spatial or contextual information related to 
the pixel of interest. Since higher resolution satellite imagery is available, it could be used to produce very accurate classifications [13]. Researchers have generally observed that when pixel-based methods are applied to high-resolution satellite images a "salt and pepper" effect was produced that contributed to the inaccuracy of the classification [4]. Thus object-based classification seems to produce better results when applied to higher resolutions.

There exist computer software packages such as eCognition and Feature Analyst that have been developed to utilize object-based classification procedures. These packages analyse both the spectral and spatial/contextual properties of pixels and use a segmentation process and iterative learning algorithms to achieve a semi-automatic classification procedure that promises to be more accurate than traditional pixel-based methods [3].

The concept of object-based image analysis as an alternative to pixel-based analysis was introduced in 1970s [11]. The initial practical application was towards automation of linear feature extraction. In addition to the limitation from hardware, software, poor resolution of images and interpretation theories, the early application of object-based image analysis faced obstacles in information fusing, classification validation, reasonable efficiency attaining, and analysis automation [9]. Since the mid-1990s, hardware capability has increased dramatically and high spatial resolution images [9] with increased spectral variability became available. Pixel-based image classification encountered serious problems in dealing with high spatial resolution images and thus the demand for object-based image analysis has increased [11]. Object-based image analysis works on objects instead of single pixels. The idea to classify objects stems from the fact that most image data exhibit characteristic texture features which are neglected in conventional classifications. In the early development stage of object-based image analysis, objects were extracted from pre-defined boundaries, and the following classifications based on those extracted objects exhibited results with higher accuracy, comparing with those by pixel-based methods [7]. This technique classifying objects extracted from predefined boundaries is applicable for agriculture plots or other land cover classes with clear boundaries, while it is not suitable to the areas with no boundaries readily available, such as semi-natural areas. Image segmentation is the solution for obtaining objects in areas without pre-defined boundaries. It is a preliminary step in object-based image analysis.

Since image classification results are essential for decision making, the methods employed in deriving this results needs to be investigated. In Nigeria, the image classification technique being used is the pixel-based. Object-based method of image classification has not been explored in Nigeria before now. Probably because of its cost which makes it difficult for an individual and sometimes even for a cooperate entities to purchase the necessary data and software tools. However, within this study high resolution satellite imagery (RapidEye, $5 \mathrm{~m}$ resolution) was acquired. The object-based and the pixel-based classification were performed and they results compared.

\section{Material and Methods}

\section{The Study Area}

Eti-Osa is a Local Government Area (LGA) in the Lagos Division of Lagos State, Nigeria located within $6^{\circ} 26^{\prime} \mathrm{N}, 6^{\circ} 28^{\prime} \mathrm{N}$ and $3^{\circ} 26^{\prime} \mathrm{E}, 3^{\circ} 32^{\prime} \mathrm{E}$. Eti-Osa LGA maintains its Eastern boundary with Ibeju-Lekki LGA and its Western boundary with Lagos Island LGA where the Eti-Osa 
LGA was created from and was known then as the Lagos City Council. It also has its Northern boundary with the Lagoon and its Southern boundary with the Atlantic Ocean. Eti-Osa LGA has a population of 283,791 , which represents $3.11 \%$ of the state's population. 158,858 of the total population are male while the remaining 124,933 are female.

\section{Image Data}

RapidEye satellite imagery data acquired in 2009 covering part of Eti-Osa LGA, Lagos State, Nigeria was procured. The sensor type used in acquiring this imagery is the multi-spectral push broom imager and is captures five spectral bands. These are: blue $(440-510 \mathrm{~nm})$, green $(520-590 \mathrm{~nm})$, red $(600-700 \mathrm{~nm})$, Red-Edge $(690-730 \mathrm{~nm})$ and near-infrared bands $(760-$ $850 \mathrm{~nm})$. It also has a panchromatic band of $1 \mathrm{~m}$. The ground sampling distance at nadir is $6.5 \mathrm{~m}$ and the orthorectified pixel size is $5 \mathrm{~m}$ with a swath width of $77 \mathrm{~km}$ [6]. Ground co-ordinates of points within the study area were obtained using handheld GPS receiver, and were used to both facilitate classification and carry out accuracy assessment.

\section{Data Processing}

ERDAS Imagine 2014 software was used in the pre-processing, pixel-based classification, and post processing of the RapidEye satellite imagery covering the study area.

For the pixel-based classification, the satellite imagery was classified by pixel-based spectral angle mapper (SAM) classifier. The signature file was generated and this involves the training of classes. AOI (Areas of Interest) was created and used to train the land cover classes (waterbody, bare-soil, vegetation and built-up) for every class, random samples were taken across the study area based on pixel spectra. The SAM Algorithm which is a supervised approach was then applied. The Spectral Angle Mapper (SAM) algorithm is based on the assumption that a single pixel of remote sensing images represents one certain ground cover material, which can be uniquely assigned to only one ground cover class. This algorithm is based on the measurement of the spectral similarity between two spectra. The spectral similarity can be obtained by considering each spectrum as a vector in $\mathrm{q}$-dimensional space, where $\mathrm{q}$ is the number of bands $[15,16]$.

The eCognition Developer was used for the object-based classification of the RapidEye satellite imagery. The extracted individual bands of the RapidEye scene acquired were stacked together into a single multispectral image using ERDAS Imagine. ArcGIS 10.1 was used to extract the shapefile of the study area from the digitized administrative map of Lagos state and to produce the land cover map of the study area. The boundary shape file (.shp) of Eti-Osa LGA was converted to an area of interest file (.aoi) which was used in sub-setting or clipping the stacked multispectral RapidEye imageries.

For the object-based image classification, the image was divided into objects serving as building blocks for further analysis using the multi resolution segmentation algorithm in eCognition software [1]. The segmentation was performed to group contiguous pixels into areas or segments that are homogenous and the following criteria were used: Scale: 450 Shape: 0.3 and Compactness: 0.5. A pair of neighbouring image objects was merged into one large object. This decision is made with local homogeneity attributes and can be defined by equation 1 [18]. 


$$
f=\sum_{i=1}^{i} W_{i}\left({ }^{n} \text { Merge } \sigma \text { Merge }-\left({ }^{n} \operatorname{Obj} 1 \sigma O b j 1+{ }^{n} \operatorname{Obj} 2 \sigma O b j 2\right)\right)
$$

Where $n$ is the number of bands and $W_{i}$ is the weight for the current band, ${ }^{n}$ Merge, ${ }^{n}$ Obj 1 and ${ }^{n} \mathrm{Obj} 2$ are respectively the number of pixels within merged object, initial object 1 , and initial object 2. Symbols $\sigma$ Merge, $\sigma O b j 1, \sigma O b j 2$ are the variances of merged object, initial object 1 , and initial object 2 is the derived local tone heterogeneity weighted by the size of image objects and summed over $n$ image bands.

Once an image had been segmented, it was then classified at the segment level which is termed object-based classification. The criteria or attributes mentioned above were used to label the objects and were used further in the object-based nearest neighbour (NN) classification. It is a supervised classification technique that classified all objects in the entire image based on the selected samples and the defined statistics.

\section{Accuracy Assessment}

The results of the pixel-based and the object-based classification of the RapidEye image were compared and their accuracy was assessed using 250 randomly generated reference points for the image. The reference data were derived from the panchromatic band of the RapidEye image for the study area. Then error matrices were generated and the assessment indices are derived, including the producer's accuracy, the user's accuracy, and the kappa statistics. To determine if the two classifications were significantly different at $(\alpha=0.05)$, a Kappa analysis and pair-wise Z-test were computed [5, 19].

$$
\begin{aligned}
\widehat{K} & =\frac{P_{0}-P_{c}}{1-P_{c}} \\
Z & =\frac{\left|\widehat{K}_{1}-\widehat{K}_{2}\right|}{\sqrt{\operatorname{var}\left(\widehat{K}_{1}\right)+\operatorname{var}\left(\widehat{K}_{2}\right)}}
\end{aligned}
$$

Where $P_{o}$ represents actual agreement which is simply the number of instances that were classified correctly throughout the entire error matrix, $P_{c}$ represents "chance agreement", which is the accuracy the classifier would be expected to achieve based on the error matrix. $\mathrm{Pc}$ is directly related to the number of each class, along with the number of instances that the classifier agreed with the ground truth class and $\widehat{K}_{1}, \widehat{K}_{2}$ represents the Kappa coefficients for the two classifications, respectively. The Kappa coefficient is a measure of the agreement between observed and predicted values and whether that agreement is by chance [19].

\section{Results and Analysis}

\section{A. RapidEye Colour Composite Imageries}

Figure 1 shows the clipped RapidEye imagery of Eti-Osa LGA using the standard "true colour" composite- bands 3, 2 and 1. Because the visible bands are used in this combination, ground features appear in colours similar to their appearance to the human visual system, healthy vegetation is green, roads are grey, and shorelines are white. 


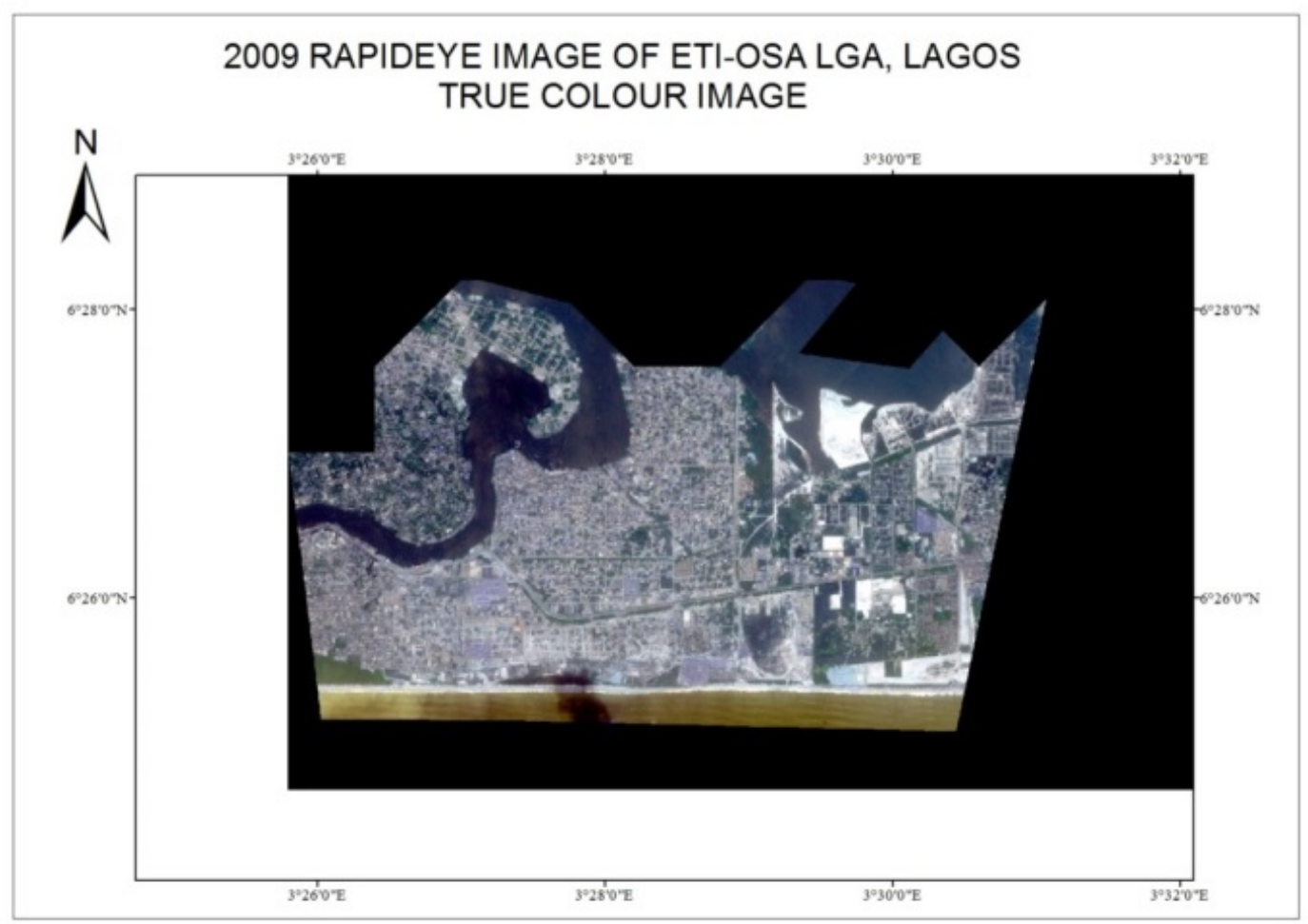

Figure 1: Composite of 2009 RapidEye Satellite Imagery

\section{B. Land Cover Maps}

The land cover maps of the study area produced for the different classification types are shown in Figures 2 and 3. Water bodies within the study area were depicted in colour blue while vegetation cover within the study area was depicted with green. The classes which are depicted in colour red represent built-up and bare soil is depicted with grey within Eti-Osa, Lagos.

\section{Accuracy Assessment}

The diagonal elements of the error matrix indicate the correctly classified pixels, while the off diagonal elements of the matrix indicate the wrongly classified pixels based on the comparison of the panchromatic band of the image, data derived from the field and the classified image. Table 1, gives the meaning of the code used in the subsequent Tables.

Table 1: Code used in Accuracy Report Tables

\begin{tabular}{|c|c|}
\hline Code & Meaning \\
\hline \hline WB & water body \\
\hline VG & vegetation \\
\hline BS & bare soil \\
\hline BU & built-up \\
\hline
\end{tabular}




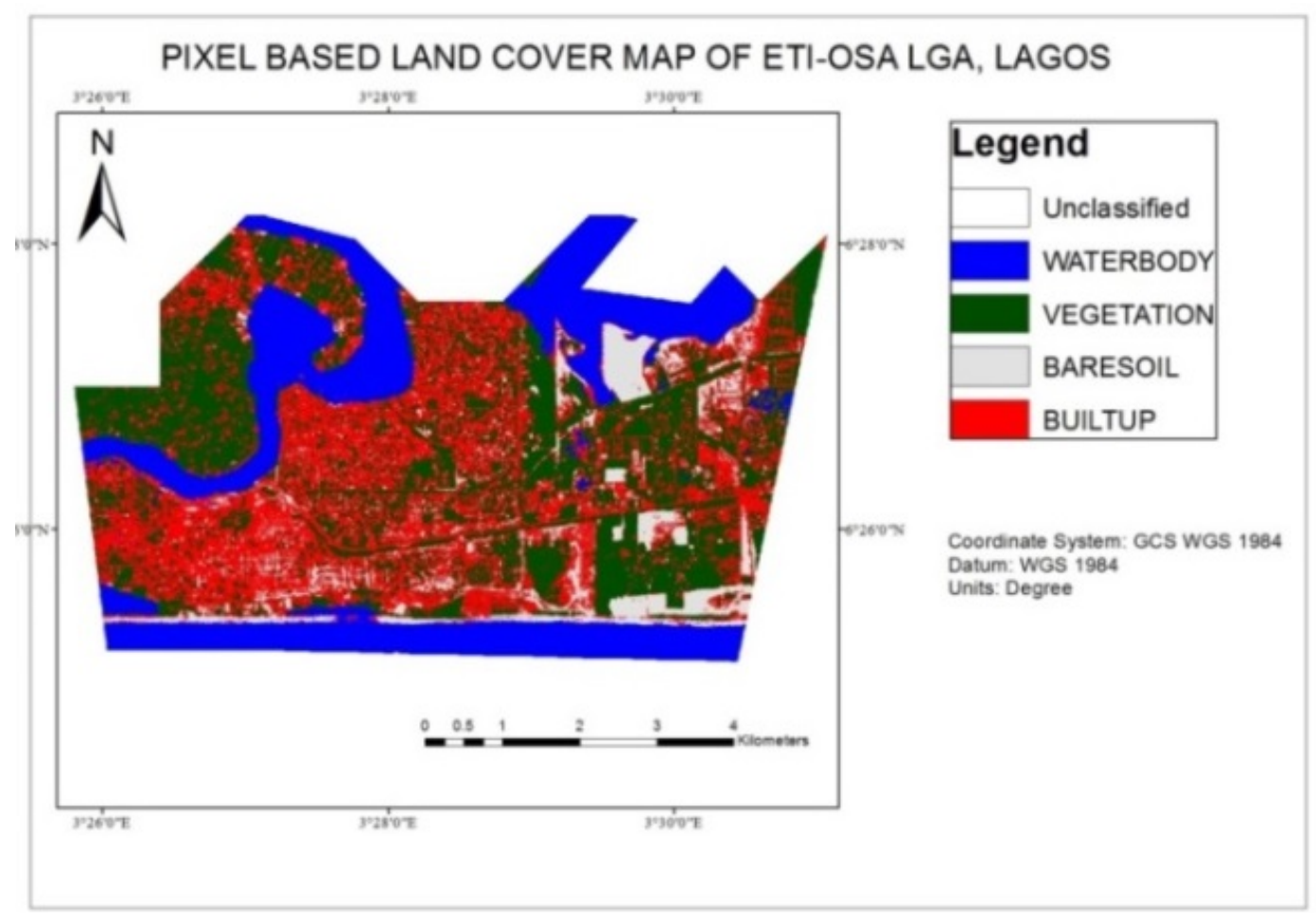

Figure 2: Land Cover Map of the Study Area (Pixel-based Classification)

OBJECT BASED LAND COVER MAP OF ETI-OSA LGA, LAGOS

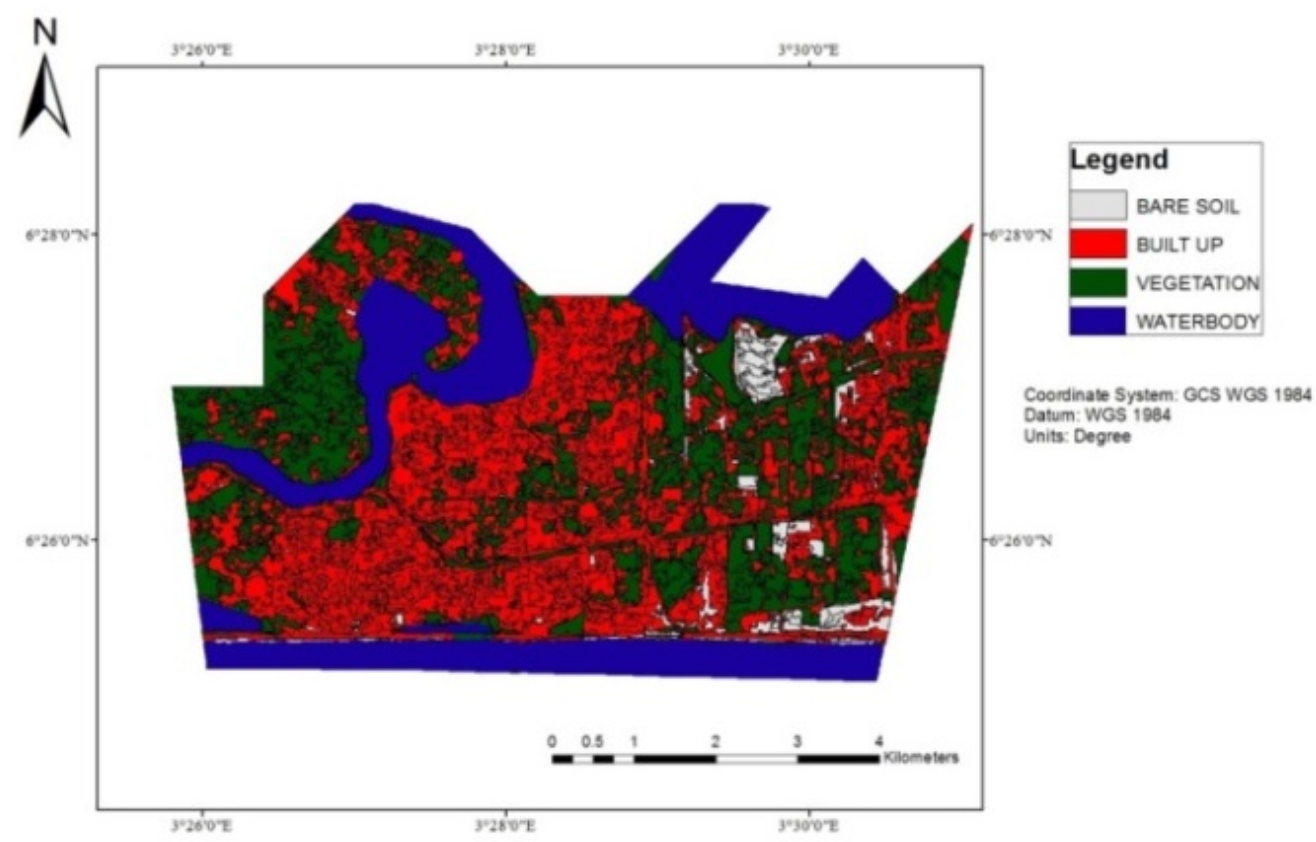

Figure 3: Land Cover Map of the Study Area (Object-based Classification) 


\section{Accuracy Report}

The results of the pixel-based and the object-oriented classification of the RapidEye image are compared by accuracy assessment. A total of the 217 samples were selected randomly for assessment. "Known" pixels from ground trothing were identified on the panchromatic band used as the reference data. Then an error matrix was generated and the assessment indices are given on Tables 2-3, including the producer's accuracy, the user's accuracy, and the kappa statistics. An accuracy assessment was also performed on the object-based classification results. The best classification result shows statistics of the training These statistics allow one to compare which classes have been best classified. The result showed that water bodies had the highest accuracy for object-based and pixel-based classification.

Table 2: Error Matrix and Accuracy Report (Pixel-based Classification)

\begin{tabular}{|c|c|c|c|c|c|c|c|}
\hline Reference Data \\
\hline $\begin{array}{c}\text { Classified } \\
\text { Data }\end{array}$ & WB & VG & BS & BU & Total & $\begin{array}{c}\text { Producer } \\
\text { Accuracy }\end{array}$ & $\begin{array}{c}\text { Users } \\
\text { Accuracy }\end{array}$ \\
\hline \hline WB & 57 & 0 & 1 & 0 & 58 & $100 \%$ & $98.28 \%$ \\
\hline VG & 0 & 58 & 0 & 11 & 69 & $89.23 \%$ & $84.06 \%$ \\
\hline BS & 0 & 0 & 19 & 3 & 22 & $70.37 \%$ & $86.36 \%$ \\
\hline BU & 0 & 7 & 7 & 54 & 68 & $79.41 \%$ & $79.41 \%$ \\
\hline Total & 57 & 65 & 27 & 68 & 217 & \\
\hline
\end{tabular}

Table 3: Error Matrix and Accuracy Report (Object-based Classification)

\begin{tabular}{|c|c|c|c|c|c|c|c|}
\hline Reference Data \\
\hline $\begin{array}{c}\text { Classified } \\
\text { Data }\end{array}$ & WB & VG & BS & BU & Total & $\begin{array}{c}\text { Producer } \\
\text { Accuracy }\end{array}$ & $\begin{array}{c}\text { Users } \\
\text { Accuracy }\end{array}$ \\
\hline \hline WB & 58 & 1 & 0 & 0 & 59 & $100 \%$ & $98.31 \%$ \\
\hline VG & 1 & 60 & 3 & 1 & 65 & $95.24 \%$ & $92.31 \%$ \\
\hline BS & 0 & 0 & 39 & 1 & 40 & $97.50 \%$ & $86.67 \%$ \\
\hline BU & 0 & 2 & 3 & 48 & 53 & $96.00 \%$ & $90.57 \%$ \\
\hline Total & 59 & 63 & 45 & 50 & 217 & \\
\hline
\end{tabular}

Kappa is a discrete multivariate technique that tests whether one data set is significantly different from another. It is used to test whether two error matrices are significantly different [5]. The two error matrices can be from different classifications, as might be the case when conducting change detection, or Kappa may be used on only one error matrix by comparing that error matrix to a hypothetical completely random error matrix. In other words, Kappa's associated test statistic KHAT tests how a classification performed relative to a hypothetical completely randomly determined classification. An important property of Kappa is that it uses the information contained in all of the cells of the error matrix, rather than only the diagonal elements, to estimate the accuracy of the classification [10]. The KHAT statistic ranges from 0 to 1 . A KHAT value of 0.75 means that the classification accounts for $75 \%$ more of the variation in the data than would a hypothetical completely random classification. 
A general framework for interpreting KHAT values was introduced by [10, 14]. They recommended that KHAT values greater than 0.8 represent strong agreement, values between 0.4 and 0.8 represent moderate agreement, and values below 0.4 represent poor agreement [10]. The Tables below show the kappa statistics for the two methods of classification employed.

Table 4: Kappa Statistics (Pixel-based Classification)

\begin{tabular}{|l|c|}
\hline \multicolumn{1}{|c|}{ Class Name } & Kappa \\
\hline \hline Waterbody & 0.9766 \\
\hline Vegetation & 0.7724 \\
\hline Bare Soil & 0.8443 \\
\hline Built-up & 0.8443 \\
\hline Overall Kappa Statistics $=0.8153$ \\
\hline
\end{tabular}

Table 5: Kappa Statistics (Object-based Classification)

\begin{tabular}{|l|r|}
\hline \multicolumn{1}{|c|}{ Class Name } & Kappa \\
\hline \hline Waterbody & 0.9842 \\
\hline Vegetation & 0.8200 \\
\hline Bare Soil & 0.8962 \\
\hline Built-up & 0.9235 \\
\hline \multicolumn{2}{|c|}{ Overall Kappa Statistics $=0.8674$} \\
\hline
\end{tabular}

\section{Comparison of Pixel-Based and Object-Based Classification}

From the Tables, it can be seen that the object-oriented classification produced more accurate results, the overall accuracy are $7.83 \%$ more than the pixel-based classification. Moreover, in the case of the pixel-based classification due to utilization of only spectral information of pixels in image data, the results looks like pepper-and-salt picture.

\section{i. Representation of Land Cover Classes by Pixels}

The Table 6 is a matrix of the number of pixels that were classified per land cover class for each of the two methods. In this Table, the numbers of pixel belonging to the same class of classified were compared.

Table 6: Matrix of Classified Pixels

\begin{tabular}{|l|l|l|}
\hline \multicolumn{3}{|l|}{ Number of Pixels } \\
\hline Land Cover Class & Pixel-Based & Object-Based \\
\hline \hline Bare Soil & 7007 & 1290 \\
\hline Built-up & 24984 & 6260 \\
\hline Vegetation & 25534 & 3910 \\
\hline Water Body & 17936 & 1380 \\
\hline
\end{tabular}

Geoinformatics FCE CTU 15(2), 2016 


\section{ii. Similarities and Differences Based on the Classified Pixels}

Table 6 shows that the comparison between pixel-based and object-based classification is possible and that the results of the two classifiers follow a general trend. However, the results from the object-based classification show rather low number of classified pixels; this is because in the case of the object-based classification, pixels have been grouped in the process of segmentation into objects. Table 6 indicates that dominant land cover within the study area is the vegetation land cover. This is followed by built-up land cover class. These results are clearly displayed by both the object-based and the pixel-based classification; however, in all cases the pixel-based classification identified more pixels than the object-based classification.

\section{Discussion}

Pixel-based and object-based image classification methods have their own advantages and disadvantages depending upon their area of application and most importantly the remote sensing datasets that are used for information extraction [9]. Traditional pixel-based classification makes use of combined spectral responses from all training pixels for a given class. Hence, the resulting signature comprises responses from a group of different land covers in the training samples. Thus, the classification system for pixel-based ignores the effect of mixed pixels [12]. However, the object-based classification uses the nearest neighbour classification (NN classification) technique because intelligent image objects are used with multi-resolution segmentation in combination with supervised classification. Pixel-based classification approach has many disadvantages when compared to object-based classification, especially in high resolution satellite data processing. Though proved to be highly successful with low to moderate spatial resolution data, pixel-based classification produces quite a lot unsatisfactory classification accuracy results with high resolution images. The use of spatial information from neighborhood or adjacent pixels remains a critical drawback to pixel-based image classification.

Object-based classification approach covers the drawbacks of pixel-based classification approach and results in outstanding classification accuracies $(7.83 \%$ higher overall accuracy than the pixel-based approach in our test). This is consistent with other studies that have shown object-based methods performs better than the pixel-based methods when applied to high resolution satellite images $[8,17]$. The object-based approach provided a significantly higher user's accuracy in the built up land cover category with an increase of $11.16 \%$. This was largely due to the better differentiation between the built up class and vegetation class using the object-based approach [13]. The bare soil land cover class yielded similar accuracies using both the pixel-based and object-based approaches, demonstrating that both types of classification methods may be beneficial to land managers and researchers interested in studying them. Object-based classification can use not only spectral information of land types, but also use pixels' spatial position, shape characteristics, texture parameters and the relationship between contexts, which effectively avoid the "salt \& pepper phenomenon" and greatly improve the accuracy of classification.

After undertaking adequate literature survey, it can be observed that for high resolution satellite image classification, object-based classification approach is considered the most suitable approach by most of the researchers as compared to pixel-based classification. The traditional pixel-based classification cannot make the best use of the relationship between pixel 
and pixels around it, which makes the classification results, become incoherent. In almost all the case studies, object-based classification approach resulted in greater accuracy ranging from $84 \%$ to $89 \%$ (approximately).

\section{Conclusion}

In this research, pixel-based and object-based image classification was performed on RapidEye satellite imagery with a $6.5 \mathrm{~m}$ spatial resolution. The image was classified by pixel-based spectral angle mapper classifier, and object-based nearest neighbour classifier, respectively. Accuracy assessment results showed that object-based image classification obtained higher accuracy than pixel-based classification. This study showed that the object-based image classification has advantage over the pixel-based classification for high spatial resolution images. The object-based method is recommended as an image classification method for high resolution images given its superiority in terms of appearance and statistical accuracy as compared to the pixel-based method. This report has only investigated the object-based method of image classification on RapidEye satellite image; it has not made an assessment on other high resolution images. It is therefore recommended that other high resolution imageries should be assessed.

\section{Acknowledgements}

We appreciate Prof. Ayobami T. Salami, the Head of Space Applications and Environmental Science Laboratory (SPAEL), Obafemi Awolowo University, Ile-Ife, Osun State, Nigeria for the imagery.

\section{References}

[1] Martin Baatz and Arno Schäpe. "Object-oriented and multi-scale image analysis in semantic networks". In: 2nd international symposium: operationalization of remote sensing. Vol. 16. 20. 1999, pp. 7-13.

[2] Thomas Blaschke et al. "Object-oriented image processing in an integrated GIS/remote sensing environment and perspectives for environmental applications". In: Environmental information for planning, politics and the public 2 (2000), pp. 555-570.

[3] J. S. Blundell and D. W. Opitz. "Object recognition and feature extraction from imagery: The Feature Analyst ${ }^{\circledR}$ approach". In: International Archives of Photogrammetry, Remote Sensing and Spatial Information Sciences 36.4 (2006), p. C42.

[4] Manuel Lameiras Campagnolo and J. Orestes Cerdeira. "Contextual classification of remotely sensed images with integer linear programming". In: CompIMAGE. 2006, pp. $123-128$.

[5] Russell G. Congalton and Kass Green. Assessing the accuracy of remotely sensed data: principles and practices. Boca Raton, Florida: CRC press, 1999, p. 137.

[6] Satellite Imaging Corporation. RapidEye Satellite Sensor. 2015. URL: http: / /www . satimagingcorp.com/satellite-sensors/other-satellite-sensors/rapideye/.

[7] A. M Dean and G. M. Smith. "An evaluation of per-parcel land cover mapping using maximum likelihood class probabilities". In: International Journal of Remote Sensing 24.14 (2003), pp. 2905-2920. DOI: 10.1080/01431160210155910. 
[8] Donald I. M. Enderle and Robert C. Weih Jr. "Integrating supervised and unsupervised classification methods to develop a more accurate land cover classification". In: Journal of the Arkansas Academy of Science 59 (2005), pp. 65-73.

[9] David Flanders, Mryka Hall-Beyer, and Joan Pereverzoff. "Preliminary evaluation of eCognition object-based software for cut block delineation and feature extraction". In: Canadian Journal of Remote Sensing 29.4 (2003), pp. 441-452. DOI: 10.5589/m03-006.

[10] Giles M. Foody. "Status of land cover classification accuracy assessment". In: Remote sensing of environment 80.1 (2002), pp. 185-201.

[11] Yan Gao and Jean Francois Mas. "A comparison of the performance of pixel-based and object-based classifications over images with various spatial resolutions". In: Online Journal of Earth Sciences 2.1 (2008), pp. 27-35. URL: http://docsdrive.com/pdfs/ medwelljournals/ojesci/2008/27-35.pdf.

[12] Yan Gao and Jean Francois Mas. "A comparison of the performance of pixel-based and object-based classifications over images with various spatial resolutions". In: International Archives of Photogrammetry, Remote Sensing and Spatial Information Sciences XXXVIII-4/C1 (2008).

[13] Steven M. de Jong, Tom Hornstra, and Hans-Gerd Maas. "An integrated spatial and spectral approach to the classification of Mediterranean land cover types: the SSC method". In: International Journal of Applied Earth Observation and Geoinformation 3.2 (2001), pp. 176-183.

[14] J. Richard Landis and Gary G. Koch. "The measurement of observer agreement for categorical data". In: biometrics (1977), pp. 159-174.

[15] Xiaofang Liu and Chun Yang. "A kernel spectral angle mapper algorithm for remote sensing image classification". In: Image and Signal Processing (CISP), 2013 6th International Congress on. Vol. 2. IEEE. 2013, pp. 814-818.

[16] S. Rashmi, S. Addamani, and S. Ravikiran. "Spectral Angle Mapper Algorithm for Remote Sensing Image Classification". In: IJISET-International Journal of Innovative Science, Engineering 83 Technology 50.4 (2014), pp. 201-205.

[17] N. D. Riggan Jr. and R. C. Weih Jr. "A comparison of pixel-based versus object-based land use/land cover classification methodologies". In: Journal of the Arkansas Academy of Science 63 (2009), pp. 145-152.

[18] L. Wang, W. P. Sousa, and P. Gong. "Integration of object-based and pixel-based classification for mapping mangroves with IKONOS imagery". In: International Journal of Remote Sensing 25.24 (2004), pp. 5655-5668.

[19] Jerrold B. Zar. Biostatistical Analysis. 4th. Upper Saddle River, New Jersey: Prentice Hall, 2007, p. 663. 
